\title{
Implementation of Noise Reduction Methodology to Modal Distribution Method
}

\author{
MyoungKeun Choi* \\ *FloaTEC, LLC, Houston, TX, USA
}

KEY WORDS: Structrual health monitoring, Vortex induced vibration (VIV), Riser, Modal distribution method, Noise reduction

\begin{abstract}
Vibration-based Structural Health Monitoring (SHM) systems use field measurements of operational signals, which are distorted by noise from many sources. Reducing this noise allows a more accurate assessment of the original "clean" signal and improves analysis results. The implementation of a noise reduction methodology for the Modal Distribution Method (MDM) is reported here. The spectral subtraction method is a popular broadband noise reduction technique used in speech signal processing. Its basic principle is to subtract the magnitude of the noise from the total noisy signal in the frequency domain. The underlying assumption of the method is that noise is additive and uncorrelated with the signal. In speech signal processing, noise can be measured when there is no signal. In the MDM, however, the magnitude of the noise profile can be estimated only from the magnitude of the Power Spectral Density (PSD) at higher frequencies than the frequency range of the true signal associated with structural vibrations under the additional assumption of white noise. The implementation of the spectral subtraction method to MDM may decrease the energy of the individual mode. In this work, a modification of the spectral subtraction method is introduced that enables the conservation of the energies of individual modes. The main difference is that any (negative) bars with a height below zero after subtraction are set to the absolute value of their height. Both noise reduction methods are implemented in the MDM, and an application example is presented that demonstrates its effectiveness when used with a signal corrupted by noise.
\end{abstract}

\section{Introduction}

Modal distribution method (MDM) is a new vibration-based Structural health monitoring (SHM) and applied to clean signal (Choi, 2009; Sweetman and Choi, 2009; 2006). However, it is unavoidable that field measurements of operational signals are distorted by noise from many sources including the sensors themselves, electromagnetic interference, wiring problems, and environmental conditions. Reducing this noise allows a more accurate assessment of the original "clean" signal and improves analysis results. Noise reduction is the process of extracting the original clean signal from a measured noisy signal, and is a popular research topic in the areas of speech signal processing and image processing.

Among numerous noise reduction strategies, the simplest method is to apply a series of bandpass filters. It is obviously most effective if the noise occurs at a relatively narrow and well defined range of frequencies. In case of a very broadband spectrum such as white noise, energy is distributed over a wide range of frequencies and bandpass filtering is ineffective. Many complicated methods of noise reduction in power spectra have been widely used in speech signal processing to estimate the Power spectrum density (PSD) of the original pure signal from signal corrupted by broadband noise. The spectral subtraction method (Boll, 1979) is a noise reduction technique that is popular due to its simple underlying concept and its effectiveness for enhancement of a signal that had been degraded by additive broadband noise. The underlying assumption is that the noise is uncorrelated as well as additive to the signal and the total (noisy) signal consists of the clean signal plus a spectrum of noise. The basic principle of the spectral subtraction method is to subtract magnitude of noise from the total noisy signal. After subtraction, any frequencies having negative power are set to a floor value.

The flooring of negative areas causes the spectral subtraction method to be inappropriate for use with the MDM. The incompatibility stems from the fact that the MDM uses the power associated with each mode to weight the relative importance of the individual modes in assessing the statistical significance of observed changes in the PSD. Implementing the spectral subtraction method to the MDM changes energy of the noise-reduced modal distributions. This change can dramatically affect the statistical importance when combining the contributions from individual modes. In this work, a variation on the spectral subtraction method is introduced that enables a conservation of energy of individual modes as well as total energy. The main difference is that any negative

Corresponding author MyoungKeun Choi: 6119 Marble Hollow Ln, Katy, TX77450, USA, 1-409-443-9019, myoungchoi@gmail.com Note that this study is based on the author's doctoral thesis of Texas A\&M University in 2009. 
areas (bars with a height below zero) are set to the absolute value of their height instead of constant value (generally zero) after subtraction.

The original power spectrum subtraction method and the proposed modification are mathematically outlined. Both methods are then implemented to the modal distribution method. An example is presented that demonstrates the effectiveness of noise reduction techniques.

\section{Methodology}

Noise reduction methodologies are general in concept, and can be implemented to nearly any measured signal that has been corrupted by noise. A variety of approaches have been proposed to reduce noise for speech enhancement: Wiener filtering, dynamic comb filtering, short-time spectral modification techniques, and others (Godsill and Rayner, 1998; Loizou, 2007). Here, characteristics of noisy signal is studied in the following subsection.

\subsection{Noisy signal}

Suppose that a measured discrete time-history $y_{m}$ is composed of a clean, noiseless signal $x_{m}$, additively combined with noise $n_{m}$ :

$$
y_{m}=x_{m}+n_{m}
$$

This additive relationship is conserved in Discrete fourier transform (DFT) space.

$$
Y_{k}=X_{k}+N_{k}
$$

where $Y_{k}, X_{k}$ and $N_{k}$ are DFTs of $y_{m}, x_{m}$ and $n_{m}$, respectively. Subscripts $m$ and $k$ will be omitted for convenience. These DFTs are complex, which are expressed in terms of magnitude and phase:

$$
Y=|Y| e^{i \theta_{Y}}=|Y| \cos \theta_{Y}+i|Y| \sin \theta Y
$$

Through multiplying both sides of Eq. (2) by their complex conjugates, the power spectral density (PSD) of measured signal including additive noise consists of the auto-spectrum and cross-spectrum of clean signal and noise:

$$
\begin{aligned}
& |Y|^{2}=|X|^{2}+|N|^{2}+X N^{*}+X^{*} N \\
& S_{Y}=S_{X}+S_{N}+S_{X N}+S_{N X}
\end{aligned}
$$

The PSD of the clean signal $S_{X}$ can be recovered by approximating the last three components in the Eq. (5). If the noise is uncorrelated with the signal, which is implied by its being independent, the cross spectra are theoretically zero: $S_{X N}=0$ and $S_{N X}=0$. Thus, if the signal and noise are stationary and independent, the power spectrum of the noise-corrupted signal, $S_{Y}$, is simply the sum of the power spectra of the signal and noise:

$$
S_{Y}+S_{X}+S_{N}
$$

Many noise reduction methodologies are based on this simple equation.

\subsection{Spectral power subtraction}

Among methods to reduce the effect of additive broadband noise, the spectral subtraction method is the most popular due to its robustness and simplicity. The PSD estimate of the clean signal is easily obtained by subtracting the power spectrum of the noise from that of the total observed signal, which includes noise:

$$
\widehat{S_{X}}=S_{Y}-S_{N}
$$

Though trivial in concept, this fundamental spectral power subtraction relation forms the basis for many noise reduction methods. However, application to the MDM of speech enhancement methodologies based on spectral subtraction has two shortcomings: First, it is based on the direct estimation of the short-term spectral magnitude, which may be less accurate than a long-term spectral magnitude for a steady signal. This inaccuracy may introduce distortion and large variation to the estimate of the enhanced spectrum. The second disadvantage of spectral subtraction is the possibility of negative estimates of power at specific frequencies in the enhanced spectrum. In such cases, the negative spectral components are conventionally floored to some values by linear or nonlinear methods. More complex methods to reduce the level of residual noise have been devised for cases in which the noise spectrum has been over-estimated (leading to negative areas under the power spectrum), including an early implementation by Berouti et al. (1979). In these methods, the portion of noise that is subtracted from the signal is adaptively adjusted according to the signal-to-noise ratio. The adaptive floor is commonly computed for spectral magnitude of noisy signal less than one of noise. Several implementations of this algorithm are presented in the literature (Berouti et al., 1979; Schless and Class, 1998).

In order to apply any algorithm for noise reduction, an estimate of the noise must first be established. In speech signal processing, the noise can be well-approximated by measurement of the signal at times when no voice is present. Unfortunately, in structural health monitoring of civil structures, the system cannot be restrained to a no-response condition such that the noise can be estimated. Instead, it is here proposed that a white-noise power spectrum be applied and the level of the white noise be approximated by the level 
detected outside the range of frequencies in which any structural response is expected or observed under white noise assumption. However the noise is estimated, there is generally some difference between the true noise and the estimated noise in both magnitude and phase. This difference can result in negative offsets in the power spectrum. Since the offset of the power spectrum is computed as a real magnitude squared, these negative offsets are not physical. Any spectral subtraction method must appropriately handle these negative densities. The simplest, though non-conservative, solution is to set any negative values to zero.

\subsection{Modified spectral subtraction methodology}

The new methodology presented here overcomes the main limitation of the spectral subtraction method: conservation of energy of individual modes. The reason that power spectrum of cleaned signal has negative bar is investigated. Phase difference between original signal, noise, and noisy signal in real part of Eq. (2).

$$
|Y| \sin \theta_{Y}=|X| \sin \theta_{X}+|N| \sin \theta_{N}
$$

where $\theta_{Y}=\theta_{X}+\phi_{Y}$ and $\theta_{N}=\theta_{X}+\phi_{N}$

$\phi_{Y}$ is phase difference between noisy signal and clean signal and $\phi_{N}$ is phase difference between clean signal and noise. Real part of Eq. (4) is expressed.

$$
|Y|^{2}=|X|^{2}+|N|^{2}+2|X||N| \cos \phi_{N}
$$

If $|X|^{2}$ is estimated by $\left.\left|\widehat{\left.X\right|^{2}}=\right| Y\right|^{2}-|N|^{2}$, then the difference between actual spectrum and estimated spectrum of the signal results from Eq. (8):

$$
\left.\left|\widehat{\left.X\right|^{2}}=\right| X\right|^{2}+2|X||N| \cos \phi_{N}
$$

If the phase difference between the noise and clean signal $\phi_{N}$ causes the cosine to be negative, it will cause the estimated magnitude of that frequency component of the signal power spectrum to be smaller than actual (clean) magnitude. For a single frequency with a relatively high noise component $(|N|)$, the magnitude of $|X|^{2}$ can be negative. The worst case happens when $|N|$ is large and the phase difference between the noise and signal is $-\pi$.

Proposed modification is taking the absolute value of any negative component, which can contribute to the total power (area under the PSD). An individual frequency component of the power spectrum is in general directly proportional to the square of the Fourier amplitude computed from the timeseries. Computing the Fourier amplitude, therefore, from a negative PSD component would seem to imply an imaginary Fourier amplitude. The PSD component is of course computed as proportional to the square of the magnitude of the Fourier amplitude, which would be equivalent to taking the absolute value of any negative PSD component. Expressing this absolute value process in equation form, the signal power spectrum estimated from the noisy spectrum is simply:

$$
\widehat{S_{X}}=\left|S_{Y}-S_{N}\right|
$$

This modification conserve energy of the PSD.

\section{Example}

In this section, original and newly proposed noise reduction methodology is implemented to the parametric MDM. Its application to a noisy signal is compared with that of the original parametric MDM. In this example, the signal and noise are simulated separately based on pre-specified target power spectra, with phase randomly drawn from a uniform distribution. The resulting realizations of signal and noise are added point-by-point in the time domain to construct a noisy signal. Here, the noise level is detected from a region of the power spectrum above the frequency range of the clean signal and that estimate is used to reduce the noise using this newly proposed methodology.

\subsection{Simulations}

Target power spectra of both signal and noise are shown in Fig. 1. The target power spectrum of signal is bi-modal and has been estimated from real measurements of vortex induced vibration of a marine riser. A white-noise power spectrum is used to simulate noise for addition to the signal over the frequency range from 0.0 to $0.7 \mathrm{~Hz}$, which is larger than the range of signal: 0.0 to $0.4 \mathrm{~Hz}$. An alternative case used to evaluate the effectiveness of the method for detection of a known frequency shift is generated separately. The signal

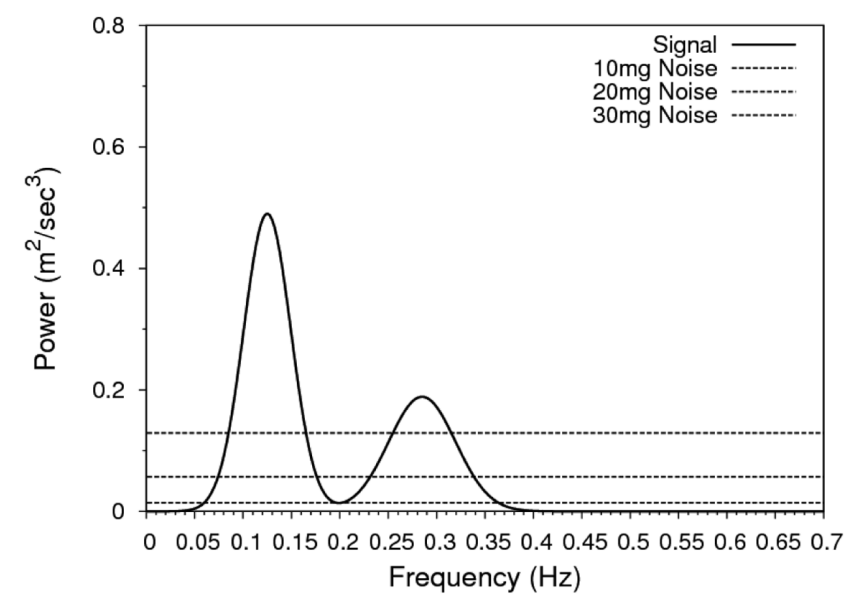

Fig. 1 Comparison of target signal and noise spectra 
Table 1 Target spectra parameters with percent change from original idealized spectrum (Choi, 2009)

\begin{tabular}{ccccc}
\hline \hline \multirow{2}{*}{$\begin{array}{c}\text { Target } \\
\text { spectrum }\end{array}$} & \multicolumn{4}{c}{ 1st Mode } \\
\cline { 2 - 5 } & Mean & Change & Variance & Change \\
\hline S & 0.125 & N/A & 0.0006 & N/A \\
S3 & 0.11879 & -0.0496 & 0.00066 & 0.1 \\
\hline Target & \multicolumn{4}{c}{ 2nd Mode } \\
\cline { 2 - 5 } spectrum & Mean & Change & Variance & Change \\
\hline S & 0.285 & N/A & 0.0012 & N/A \\
S3 & 0.27622 & -0.0305 & 0.00132 & 0.1 \\
\hline
\end{tabular}

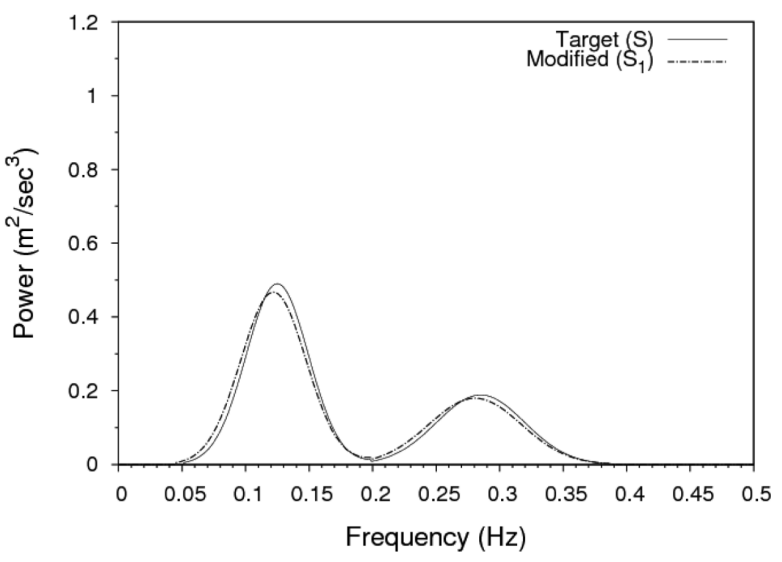

Fig. 2 Two sample target spectra (S and S1) (Choi, 2009)

power spectrum is modified as if the stiffness of the measured system has been decreased. Transforming each modal distribution of the original target spectrum (S) results in the modified target spectrum, S3, shown in Table 1. Details are fully explained in Choi (2009) and Sweetman and Choi (2009). Fig. 2 shows two target spectrum samples.

Three families of realizations based on the original target spectrum (S) of the signal or the modified spectrum (S3) of
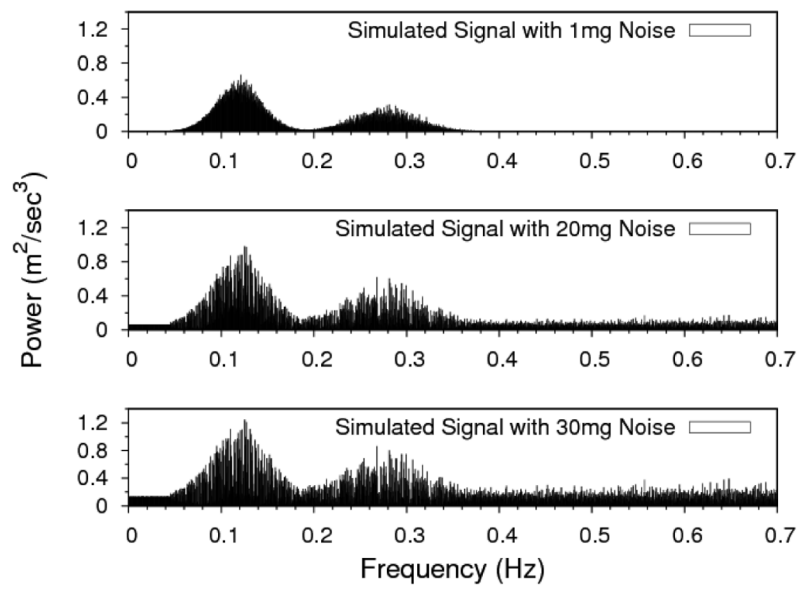

Fig. 3 Sample signal spectrums with 1, 20, and $30 \mathrm{mg}$ RMS noise the signal are corrupted by realizations of the noise spectrum with four different RMS values; 10, 20, and $30 \mathrm{mg}$. Sample power spectra of noisy signals are shown in Fig. 3. Each of tested three cases is statistically analyzed through 25 independent realizations and subsequent tests of the noise reduction methodology coupled with the MDM. All realizations have a time step of $0.2 \mathrm{sec}$.

\subsection{Application of MDM to signal corrupted by noise}

The development of the original parametric MDM is based on the assumption of well-separated Gaussian modes. Increasing levels of noise challenge both of these assumptions: the peaks become less well-separated and the distribution becomes less Gaussian. As such, the original method would not be expected to be appropriate for a signal corrupted by large amounts of noise.

The Fig. 4 shows that noise corruptions (10 to $30 \mathrm{mg}$ RMS) prevent the parametric MDM from detecting changes in underlying modal distribution of signal. Less than $5 \%$ significance level is considered as detection of change in underlying modal distributions. There is almost no decrease or increase in the significance level with increasing time duration. In all the following figures, the symbols represent the mean of 25 computed significance levels and the error bars indicate one standard deviation. Neither the significance level nor its uncertainty decreases with time duration. This trend indicates the method will be ineffective regardless of the amount of data available.

In high noise situations, the individual modes resulting from the penalty method process have very non-Gaussian shape because the would-be tails are trimmed vertically at the separation point. Thus, the most possible explanation for the break-down of the method with high noise is the resulting

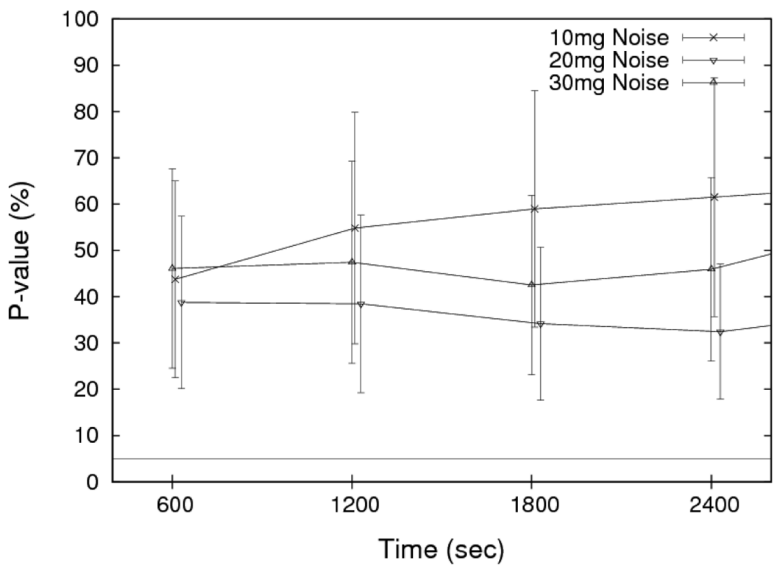

Fig. 4 Mean and standard deviation of 25 significance levels from parametric MDM applied to signals whose underlying modal distributions are changed 
underlying requirement of parametric test, the Gaussian distribution, is increasingly violated, as shown in Fig. 3.

\subsection{Application of noise reduction methodology}

Two noise reduction methods, 1) spectral subtraction method with flooring zero and 2) new method explained in section 2.3, are implemented to the MDM and applied to the same noisy signals in section 3.2. Noise reduction methodologies are applied to decrease the noise corruption before the MDM is applied. New method is applied to the sample noisy spectra (Fig. 3) and the resulting cleaned spectra is presented in Fig. 5.

Two noise reduction methods are implemented and Fig. 6 shows the resulting P-values for MDM with noise reduction methods. The probability is low and continues to drop with increasing amounts of data for both noise reduction methods. The spectral subtraction method with flooring zero produce lower P-values. This underestimated P-value is unrealistic and caused by underestimating the variance of each mode: it can be that the higher noise (somehow) enables the better signal

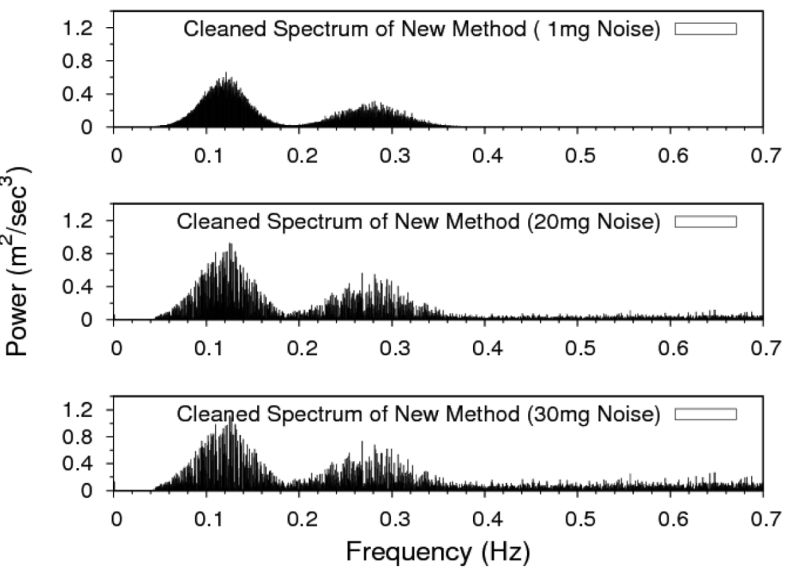

Fig. 5 Sample cleaned spectra of new noise reduction method

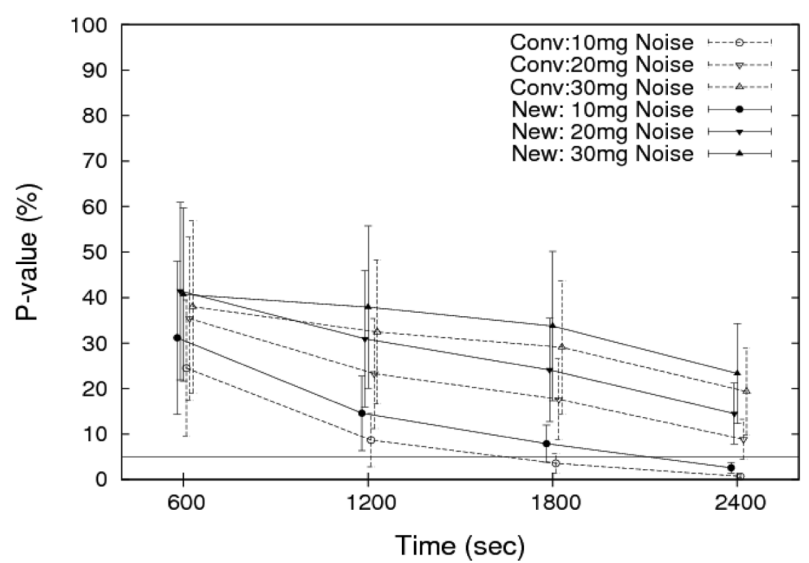

Fig. 6 Comparison of detection ability of MDMs using conventional and new noise reduction methods processing.

Figure 4 and Figure 6 show noise reduction methods markedly improves the MDM results: the computed significance level and its uncertainty are both decreased for all noise levels. In the case of $10 \mathrm{mg}$ RMS noise, it has already been overcome with application of 2,400 seconds of data. Application to higher level of noise (20 or $30 \mathrm{mg}$ RMS noise) implies that sufficient data might enable statistical detection of this change since there still remains an inverse proportional relationship between significance level and time duration.

\section{Conclusion}

Undesirable noise in field measurements is unavoidable, though it should be reduced as much as practical to minimize degradation of analysis results. Spectral subtraction methods offer simple and effective methodologies to reduce additive white noise. The modification is introduced that energy of individual modes is conserved through setting of negative bar to the absolute area. This avoids possibility of unreasonable P-value due to underestimation of variance in spectral subtraction method with flooring zero.

Without noise reduction, the original parametric MDM proved to be less effective in the presence of high noise. With very high noise, the significance level and its uncertainty are not observed to decrease with increasing time duration (Fig. 4), which implies the method will not work on this noisy data regardless of the amount of data available.

Implementation of noise reduction methodology to the parametric MDM meaningfully improves analysis results for noisy measurements. The desired inverse relationship between significance level and time duration is observed although higher noise requires longer monitoring time (Fig. 6). The method described here still requires that the shape of the peaks after noise reduction be Gaussian. Unfortunately, that requirement is increasingly poorly met as the noise level increases (Fig. 5). New methodology can be developed with nonparametric statistical comparison instead of T-statistics (parametric).

\section{References}

Berouti, M., Schwartz, R. and Makhoul, J. (1979). "Enhancement of Speech Corrupted by Acoustic Noise", Proceeding of IEEE International Conference on Acoustics, Speech, and Signal Processing, Vol 4, pp 208-211.

Boll, S. (1979). "Suppression of Acoustic Noise in Speech Using Spectral Subtraction", IEEE Transactions on Acoustics, Speech and Signal Processing, Vol 27, pp 
113-120.

Choi, M. (2009). The Modal Distribution Method: A New Statistical Algorithm for Analyzing Measured Response, Thesis (PhD), Texas A\&M University.

Godsill, S.H. and Rayner, P.J. (1998). Digital Audio Restoration: A Statistical Model Based Approach, Secaucus, NJ, USA, Springer-Verlag Inc.

Loizou, P.C. (2007). Speech Enhancement: Theory and Practice, Boca Raton, FL. CRC Press.

Schless, V. and Class, F. (1998). SNR-dependent Flooring and Noise Overestimation for Joint Application of Spectral Subtraction and Model Combination, Proceeding of the 5th
International Conference on Spoken Language Processing. Sweetman, B. and Choi, M. (2009). The Modal Distribution Method for Statistical Analysis of Measured Structural Response, Journal of Probabilistic Engineering Mechanics. Sweetman, B. and Choi, M. (2006). The Modal Distribution Method: A New Statistical Algorithm for Analyzing Measured Acceleration Data, Proceedings of SPIE, Volume 6174, page 61742H-1, San Diego, California, USA.

2011년 1월 12일 원고 접수 2011년 3월 11일 심사 완료 2011년 4월 22일 게재 확정 